\title{
Three-dimensional nonadiabatic electron- optical gyrotron systems with inhomogeneous current density
}

\author{
Kseniia Leshcheva ${ }^{1,2, *}$, Vladimir Manuilov ${ }^{1,2}$, and Arkadiy Goldenberg ${ }^{1}$ \\ ${ }^{1}$ Institute of Applied Physics of the Russian Academy of Sciences, Nizhny Novgorod, Russia \\ ${ }^{2}$ N. I. Lobachevsky State University of Nizhny Novgorod, Nizhny Novgorod, Russia
}

\begin{abstract}
The results of a numerical study of the effect of threedimensional perturbations of the space charge density distribution in a nonadiabatic electron-optic systems of gyrotrons are presented. Data of direct numerical simulation using the 3-D program CST Studio Suite are described.
\end{abstract}

Traditionally, adiabatic magnetron-injection guns (MIG) are used as electron-optical systems (EOS) of gyrotrons [1]. Along with the undoubted advantages (reliability, simplicity, low criticality), MIGs also have a number of fundamental shortcomings, among which, due to the specifics of the formation of a helical electron beam (HEB) in adiabatic fields, the most serious and fundamentally insoluble is the effect of emitter roughness and thermal initial electron velocities. These factors cause a significant increase in the spread of rotational velocities of electrons, especially in the gyrotrons of the millimeter and submillimeter wavelength ranges, which leads to the need to reduce the pitch factor (the ratio of the rotational and longitudinal velocities of the electron in the working space) to maintain beam stability. The influence of roughness and thermal velocities can be drastically reduced with a non-adiabatic HEB formation scheme, since in this case a straight-line beam is initially formed, and only then is it initially twisted in a non-adiabatic electric or magnetic field. A further increase in the oscillatory velocity of the particles to the required value is performed due to adiabatic compression of the beam in a smoothly growing magnetic field. There are several schemes of non-adiabatic EOS [2]. One of the new and recently proposed one is the axially symmetric system (fig. 1, fig. 2) [3], in which a rectilinear beam is injected through an annular gap at an angle to the magnetic field and, as a result, the primary twist of the beam occurs. The first numerical calculations of such systems showed that in them, in comparison with the MIGs, one and a half to two-fold decrease in the velocity spread can be reached and due to this a significant increase in the pitch factor maybe obtained keeping the stability of the HEB.

Electron-optical systems of gyrotrons, unlike most other microwave vacuum devices utilizing the space charge limitation emission regime, operate in the mode of temperature limitation of emission. Therefore, due to the azimuthal inhomogeneity of the cathode heating, a significant violation of the azimuthal symmetry of the distribution of the

*Corresponding author: ksenilescheva@mail.ru 
emission current density is observed. It leads to the azimuthal inhomogeneity of the electron beam. This is the reason for the appearance of inhomogeneous fields, which, at least in MIGs, in turn, increase the velocity spread of electrons, contribute to the excitation of spurious low-frequency oscillations and the appearance of the spread of electron-electron particle energy [4]. So, one can expect that inhomogeneous emission might affect the velocity distribution in non-adiabatic EOS also. Besides, it was experimentally shown in [4] that a violation of the axial symmetry of the distribution of the emission current can lead to a significant decrease in the efficiency of the gyrotron and to a decrease in the stability of the HEB. The inhomogeneous beam structure is also responsible for the development of spurious modes in the cavity [5].

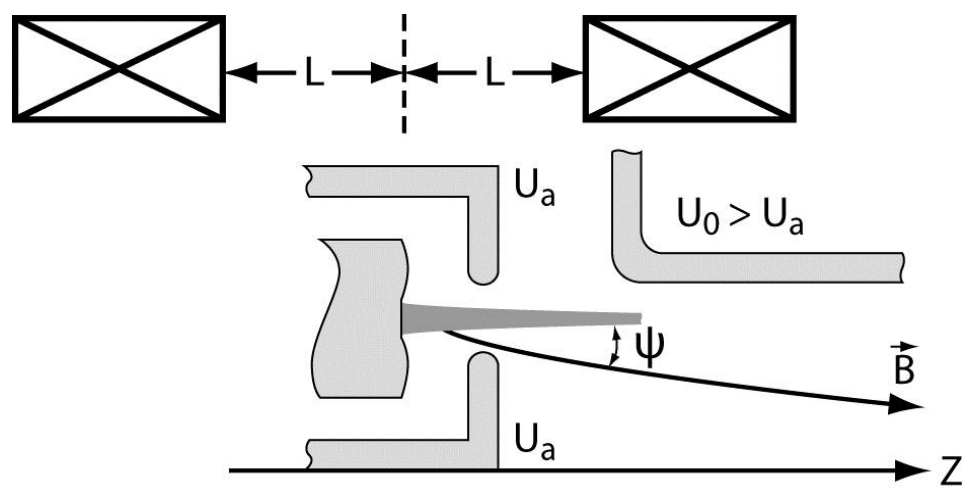

Fig. 1. Scheme of a non-adiabatic electron-optical system.

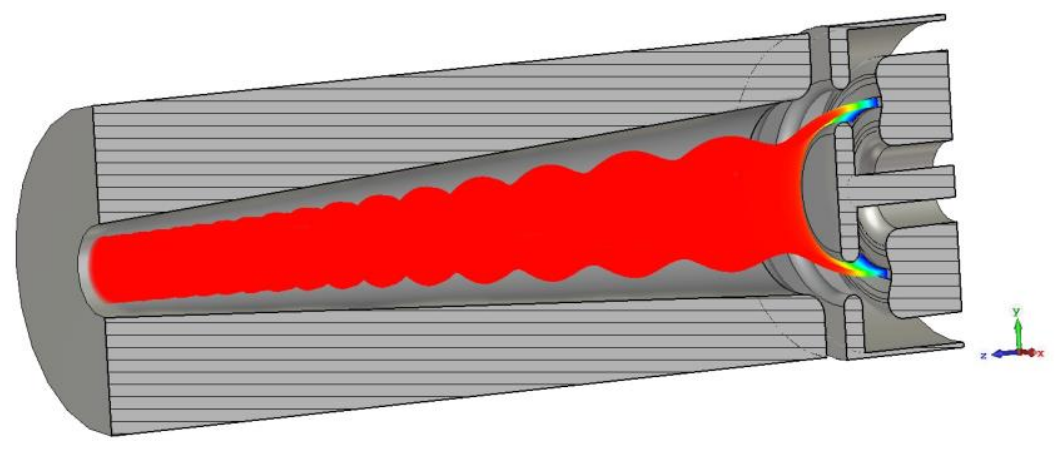

Fig. 2. Electrodes shape and electron beam position in the non-adiabatic electron-optical system.

However, at the moment, the question of the influence of emission heterogeneity on the operation of non-adiabatic EOS remains unexplored. Despite the fact that the influence of the emitter roughness and the initial thermal electron velocities on the operation of the device in non-adiabatic systems was reduced, the effect of inhomogeneous heating of the cathode continues to be an urgent issue in the theory of such systems.

The theoretical analysis of HEBs has so far been carried out mainly in the framework of two-dimensional numerical models assuming axial symmetry of the current density distribution [6-7]. Recently appeared first works devoted to the trajectory analysis of HEBs in the three-dimensional approximation [8-10] used a simplified physical model of the beam and were devoted to the study of a limited number of simplest configurations of adiabatic MIGs with broken azimuthal symmetry of the beam current. Finally, we note that the emergence of new variants of non-adiabatic formation systems [3] requires consideration of the EOS, where both the axial symmetry of some electrodes and the uniformity of the current density distribution in azimuth are violated simultaneously. 
The new developed methodology for modeling of the non-adiabatic systems is based on the joint use of the CST Studio Suite package [11] for trajectory analysis of threedimensional electron-optical systems, and special programs for pre- and post-processing, implemented in Python [12], which allow setting initial conditions for particles and special algorithms for processing the results of trajectory analysis to realize the more complicated physical model of the beam, taking into account, in addition to the space charge field, the arbitrary violation of the electron gun geometry and current density distribution at the emitter and get detailed information about the parameters of the beam as a whole or some of its azimuthal fractions at any cross-sections.

Based on the developed technique, a numerical simulation of the heterogeneity of thermionic emission in the non-adiabatic EOS was performed (Table 1). The gyrotron had the following parameters: current $\mathrm{I}=3 \mathrm{~A}$, accelerating voltage $\mathrm{U}_{0}=15 \mathrm{kV}$, operating magnetic field $\mathrm{B}_{0}=0.65 \mathrm{~T}$. Below the followingdependencies of the current density on the azimutal coordinate $\theta$ were considered homogeneous $(j=$ const $)$ and cosine distributions $\left(j=2^{*}\left[1+a^{*} \cos (2 \theta)\right]\right.$ ) (here $j$ is the current density in $\mathrm{A} / \mathrm{cm}^{2}, a$ is the modulation amplitude).

Table 1. HEB parameters calculated for different heterogeneity of thermionic emission.

\begin{tabular}{|c|c|c|}
\hline$a$ & Pitch-factor, $\mathrm{g}$ & Velocity spread $\delta \mathrm{V}_{\perp}, \%$ \\
\hline 0 & 1.608 & 13.07 \\
\hline 0.25 & 1.963 & 11.135 \\
\hline 0.50 & 1.983 & 15.878 \\
\hline 0.75 & 2.021 & 18.318 \\
\hline
\end{tabular}

According to the results of numerical simulation big modulation $(a>1)$ of the current density leads to significant increase of the velocity spread. Let us note that according to the experimental measurements [5], the inhomogeneity of the current density over azimuthal coordinate may reach $50-70 \%$ at least, depending of the technique of the emitter manufactoring. So, in experimental conditions, it is necessary to provide small enough ( $a \leq 0.5$ at least) violation of current density distribution in the non-adiabatic systems also.

The reported study was funded by RFBR according to the research project № 18-32-00142 and IAP RAS project No. 0030-2019-0019.

\section{References}

1. A.L.Goldenberg, M.I.Petelin, Radiophys. Quantum Electron., Formation of helical electron beams in adiabatic gun, v.16(1), pp. 106-111 (1973)

2. M. Yu. Glyavin, A. L. Goldenberg, V. N. Manuilov, M. V. Morozkin, Radiophys. Quantum Electron., Experimental studies of the electron-optical system of a lowvoltage gyrotron with a nonadiabatic electron gun, v.54(8-9), pp. 622-626 (2011)

3. A. L. Goldenberg, M.Yu.Glyavin, K. A. Leshcheva, V.N.Manuilov, Radiophys. Quantum Electron., Nonadiabatic electron-optical system of a technological gyrotron, v.60(5), pp. 395-400 (2017) 
4. O. Louksha, B. Pioszyk, G.Sominski et al, IEEE Trans.Plasma Science, On potentials of gyrotron efficiency en-hancement: measurements and simulations on a 4-mm gyrotron, v.34(3), pp. 502-511(2006)

5. K. A. Leshcheva and V. N. Manuilov, Usp. Prikl. Fiz., Numerical simulation of 3-D systems of formation of helical electron beams of gyro-devices with azimuthally inhomogeneous distribution of emission current, v.7 (3), pp. 298-308 (2019)

6. W.B. Hermannsfeldt, SLAC Report 226, Stanford Linear Accelerator Center, Stanford University, Electron trajectory program, p.119 (1979)

7. P.V. Krivosheev, V.K. Lygin, V.N. Manuilov, Sh.E. Tsimring, Int. J. of Infrared and MM waves, Numerical Simulation Models of Focusing Systems of Intense Gyrotron Helical Electron Beams, vol.22(8), pp. 1119-1146 (2001)

8. O.I. Luksha, P.A. Trofimov, Technical Physics, Simulation of Non-Uniform Electron Beams in the Gyrotron Electron-Optical System, 63(4), pp.598-604, (2018)

9. J. Gr. Pagonakis, J. L. Vomvoridis, IEEE Trans. Plasma Sci., Evolution of an electron beam with azimuthal density nonuniformity in a cylindrical beam tunnel, 32(3), pp. 890-898 (2004)

10. A.Malygin, S.Illy, I.Gr.Pagonakis, B.Piosczyk, S.Kern, et al., IEEE Trans. Plasma Sci., Design and 3-D simulations of a 10-kW/28-GHz gyrotron with a segmented emitter based on controlled porosity-reservoir cathodes, 41(10),pp. 2717-2723 (2013)

11. https://www.cst.com/Products/CST

12. Python Software Foundation www.python.org 